\title{
Remarks on the Henstock Integral of Stieltjes Type
}

\author{
Yôto KuBota*
}

\section{Abstract.}

Let $f:[a, b] \rightarrow \boldsymbol{R}$ be bounded and $g:[a, b] \rightarrow \boldsymbol{R}$ be of bounded variation. It is shown that $f$ is Henstock integrable with respect to $g$ on $[a, b]$ if and only if $f$ is Young refinement integrable with respect to $g$ on $[a, b]$, and both integrals have the same value. Some relations to the mean Stieltjes integral will also be given.

Let $[a, b]$ be a compact interval on the real line $\boldsymbol{R}$. Given a positive function $\delta:[a, b] \rightarrow(0, \infty)$, we consider finite sequences of numbers $A=\mid x_{0}, x_{1}, \ldots, x_{n} ; t_{1}$, $\left.t_{2}, \ldots, t_{n}\right\}$ such that

$$
\begin{aligned}
& a=x_{0}<x_{1}<\cdots<x_{n}=b, \quad x_{i-1} \leq t_{i} \leq x_{i} \\
& {\left[x_{i-1}, x_{i}\right] \subset\left(t_{i}-\delta\left(t_{i}\right), t_{i}+\delta\left(t_{i}\right)\right) \quad(i=1,2, \ldots, n) .}
\end{aligned}
$$

Such a sequence $A$ is called a $\delta$-fine partitions of $[a, b]$.

If the second condition is replaced by

$$
x_{0} \leq t_{1}<x_{1}, x_{i-1}<t_{i}<x_{i}(i=2,3, \ldots, n-1), \quad x_{n-1}<t_{n} \leq x_{n}
$$

then $A$ is called a $\delta^{*}$-fine partition of $[a, b]$.

Definition 1. A function $f:[a, b] \rightarrow \boldsymbol{R}$ is said to be $H$-intregrable, (resp. $H^{*}$-intergrable) with respect to $g:[a, b] \rightarrow \boldsymbol{R}$ on $[a, b]$ if there exists a number $\alpha$ such that to every $\varepsilon>0$ there is such a positive function $\delta(x)$ that

$$
\left|\sum_{i=1}^{n} f\left(t_{i}\right)\left(g\left(x_{i}\right)-g\left(x_{i-1}\right)\right)-\alpha\right|<\varepsilon
$$

for any $\delta$-fine partition (resp. $\delta^{*}$-fine partition) $A=\left\{x_{0}, x_{1}, \ldots, x_{n} ; t_{1}, t_{2}, \ldots, t_{n}\right\}$. The number $a$ is denoted by $(H) \int_{a}^{b} f d g\left(\left(H^{*}\right) \int_{a}^{b} f d g\right)$.

Definition 2. Let $g:[a, b] \rightarrow R$ be such that the limits $g(x+), g(x-)$ exist

Received March 2, 1985. Research partially supported by the Grant-in-Aid for Scientific Reseach C-59540065 from the Ministry of Education.

- Department of Mathematics, Ibaraki University, Mito, Ibaraki 310, Japan. 
for all $x \in[a, b]$. A function $f:[a, b] \rightarrow \boldsymbol{R}$ is said to be Young refinement integrable with respect to $g$ if there is number $\alpha$ such that to every $\varepsilon>0$ there exists a partition $P_{0}$ of $[a, b]$ in the ordinary sense so that

$$
\begin{gathered}
\mid \sum_{i=1}^{n}\left[f\left(x_{i-1}\right)\left(g\left(x_{i-1}+\right)-g\left(x_{i-1}\right)\right)+f\left(t_{i}\right)\left(g\left(x_{i}-\right)-g\left(x_{i-1}+\right)+\right.\right. \\
+f\left(x_{i}\right)\left(g\left(x_{i}\right)-g\left(x_{i}-\right)\right]-\alpha \mid<\varepsilon
\end{gathered}
$$

holds for any ordinary partition $P=\left\{a=x_{0}, x_{1}, \ldots, x_{n}=b\right\}$ with $x_{i-1}<t_{i}<x_{i}(i=1$, $2, \ldots, n)$ which is a refinement of $P_{0}$. The number $\alpha$ is denoted by $(Y) \int_{a}^{b} f d g$.

In [1] and [2] Schwabik studied the relation between the $H$ or $H^{*}$-integral and the $Y$-integral, and established the following theorem.

THEOREM 1. Let $f:[a, b] \rightarrow \boldsymbol{R}$ and let $g:[a, b] \rightarrow \boldsymbol{R}$, be of bounded uariation. The existence of $(Y) \int_{a}^{b} f d g$ dose not in general imply the existence of $(H) \int_{a}^{b} f d g$. If $(Y) \int_{a}^{b} f d g$ exists then also $\left(H^{*}\right) \int_{a}^{b} f d g$ exists and both intergrals are equal.

In this note we want to show the following theorem.

THEOREM 2. Let $f:[a, b] \rightarrow \boldsymbol{R}$ be bounded and $g:[a, b] \rightarrow \boldsymbol{R}$ be of bounded variation. Then $(Y) \int_{a}^{b} f d g$ exists if and only if $(H) \int_{a}^{b} f d g$ exists, and both integrals have the same value.

To prove Theorem 2 we need some lemmas.

LEMMA 1. If $f:[a, b] \rightarrow \boldsymbol{R}$ is bounded, $g:[a, b] \rightarrow \boldsymbol{R}$ is of bounded variation and $(Y) \int_{a}^{b} f d g$ exists then $(H) \int_{a}^{b} f d g$ exists and both integrals are eqnal.

This lemma has been proved in Theorem 3.2 in [1].

LEMMA 2. If $g:[a, b] \rightarrow \boldsymbol{R}$ is a pure break function of bouded variation and $f:[a, b] \rightarrow R$ is bounded then $(Y) \int_{a}^{b} f d g$ and $(H) \int_{a}^{b} f d g$ both exist and are equal.

For the proof, see Corollaries 1.2 and 1.2 in [1].

Lemma 3. If $f:[a, b] \rightarrow R$ is bounded, $g:[a, b] \rightarrow \boldsymbol{R}$ is continuous and of 
bouvded variation and if $(H) \int_{a}^{b} f d g$ exist then the Riemann-Stieltjes refinement integral $(R) \int_{a}^{b} f d g$ exists and both integrals are equal.

Proof. For a given $\varepsilon>0$, there is a function $\delta_{1}(x):[a, b] \rightarrow(0, \infty)$ such that

$$
\left|\sum_{i=1}^{n} f\left(t_{i}\right)\left(g\left(x_{i}\right)-g\left(x_{i-1}\right)\right)-(H) \int_{a}^{b} f d g\right|<\varepsilon
$$

for any $\delta_{1}$-fine partition $\left\{x_{0}, x_{1}, \ldots, x_{n} ; t_{1}, t_{2}, \ldots, t_{n}\right\}$ of $[a, b]$. Since $g$ is continuous and of bounded variation on $[a, b]$, its total variation on $[a, x] V_{a g}^{x} g$ is continuous on $[a, b]$. Hence there is a positive number $\delta_{2}$ such that $V_{x}^{y} g<\varepsilon$ for any $x, y \in[a$, b] with $0<y-x<\delta_{2}$. Let $\delta(x)=\min \left(\delta_{1}(x) / 2, \delta_{2}\right)$. Let $\left\{y_{0}, y_{1}, \ldots, y_{m} ; t_{1}, t_{2}, \ldots, t_{m}\right\}$ be the fixed $\delta(x)$-fine partition of $[a, b]$ and put $P_{0}=\left\{y_{0}, y_{1}, \ldots, y_{m}\right\}$. If $P$ is any refinement of $P_{0}$ and if $y_{j-1}=z_{0}<z_{1}<\cdots<z_{l j}=y_{j}$ is the corresponding part of $P$ on $\left[y_{j-1}, y_{j}\right](j=1,2, \ldots, m)$ then we have for $z_{k-1} \leq \xi_{k} \leq z_{k}\left(k=1,2, \ldots, l_{j}\right)$

$$
\begin{aligned}
& \left|\sum_{k=1}^{l j} f\left(\xi_{k}\right)\left(g\left(z_{k}\right)-g\left(z_{k-1}\right)\right)-f\left(t_{j}\right)\left(g\left(y_{j}\right)-g\left(y_{j-1}\right)\right)\right| \\
& \quad \leq \sum_{k=1}^{l j}\left|f\left(\xi_{k}\right)-f\left(t_{j}\right)\right|\left|g\left(z_{k}\right)-g\left(z_{k-1}\right)\right| \leq 2 M \sum_{k=1}^{l j}\left|g\left(z_{k}\right)-g\left(z_{k-1}\right)\right| \\
& \quad \leq 2 M V_{y_{j-1}{ }^{9 j}}^{{ }^{j}} g<2 M \varepsilon,
\end{aligned}
$$

where $|f(x)| \leq M$ on $[a, b]$.

Let $S(P, f, g)$ denote the Riemann-Steltjes sum of $f$ with respect to $g$ for $P$. Then we get

$$
\left|S(P, f, g)-\sum_{j=1}^{m} f\left(t_{j}\right)\left(g\left(y_{j}\right)-g\left(y_{j-1}\right)\right)\right|<2 M \varepsilon m
$$

and therefore

$$
\left|S(P, f, g)-(H) \int_{a}^{b} f d g\right|<2 M \varepsilon m+\varepsilon
$$

which completes the proof.

Proof of THeORem 2.

Necessary part is given in Lemma 1. To prove the sufficiency, we represent $g=g_{b}+g_{c}$ where $g_{b}$ is the pure break part and $g_{c}$ is the continuous part of $g$ respectively. Then $g_{b}, g_{c}$ are of bounded variation. It follows from Lemma 2 that $(H) \int_{a}^{b} f d g$ and $(Y) \int_{a}^{b} f d g$ exist and are equal. Hence $(H) \int_{a}^{b} f d g_{c}$ must exists since the $H$-integral is additive with respect to integrators. By Lemma 3 , 
$(R) \int_{a}^{b} f d g_{c}$ exists and equals $(H) \int_{a}^{b} f d g_{c}$. Since the $Y$-integral includes the $R$ integral in this case, we have

$$
(H) \int_{a}^{b} f d g_{c}=(Y) \int_{a}^{b} f d g_{c}
$$

Hence $(Y) \int_{a}^{b} f d g$ exists and equals $(H) \int_{a}^{b} f d g$.

Finally we shall give some remarks to the mean Stieltjes refinement integral (M) $\int_{a}^{0} f d g$ (for the definition of the $M$-integral see [4]).

REMARK 1. The $H$-integral $(H) \int_{a}^{b} f d g$ is not compatible to the $M$-integral $(M) \int_{a}^{b} f d g$, that is, there exist functions $f, g:[a, b] \rightarrow R$ such that $f$ is both $H$ and $M$-integrable with respect to $g$ on $[a, b]$ but

$$
(H) \int_{a}^{b} f d g \neq(M) \int_{a}^{b} f d g .
$$

Let $f, g$ be real valued functions defined on $[-1,1]$ as follows; $f(x)=0$ except at 0 on $[-1,1]$ and $f(0)=1: g(x)=0(-1 \leq x<0), g(0)=1 / 2$ and $g(x)=1$ $(0<x \leq 1)$. Then we have

$$
\begin{aligned}
& (M) \int_{-1}^{1} f d g=1 / 2(f(0-)+f(0))(g(0)-g(0-)) \\
& +1 / 2(f(0)+f(0+))(g(0+)-g(0)) \\
& =1 / 2 \text {, } \\
& \text { (H) } \int_{-1}^{1} f d g=0
\end{aligned}
$$

REMARK 2. Suppose $g$ is of bounded variation on $[a, b], f$ is bounded and $(M) \int_{a}^{b} f d g$ exists. Then $(H) \int_{a}^{b} f d g$ exists and equals

$$
\begin{gathered}
(M) \int_{a}^{b} f d g-1 / 2 \sum_{x \in[a, b]}(f(x+)-f(x))(g(x+)-g(x-)) \\
+1 / 2 \sum_{x \in[a, b]}(f(x)-f(x-))(g(x)-g(x-)) .
\end{gathered}
$$

It follows from Theorem 2.4 in [5] that $(Y) \int_{a}^{b} f d g$ exists and equals the above value. By Theorem $2,(H) \int_{a}^{b} f d g$ exists and has the same value. 


\section{References}

[1] S. Schwabik: On the relation between Young's and Kurzweil's concept of Stieltljes integral, Ċas. Pēst,. Mat. 98 (1973), 237-251.

[2] - On a modified sum integral of Stieltles type, ibid., 274-277.

[3] R. M. McLeod: The generalized Riemann integral, Carus Math. Monograph, No. 20, Math. Ass. Amer. 1980.

[4] T. H. Hildebrandt: Introduction to the theory of integration, Academic Press, New York, London 1963.

[5] F.M. Wright and J.D. Baker: On integration by parts for weighted integrals, Proc. Amer. Math. Soc., 22 (1969), 42-52. 